\title{
Surgical Repair of Dorsal Capsulo-Ligamentous Complex for Dislocation of the Thumb Carpometacarpal Joint
}

\author{
Haruhiko Shimura ${ }^{1}$, Tetsuya Sato ${ }^{2}$, Akimoto Nimura ${ }^{3}$, Yoshiaki Wakabayashi', Koji Fujita ${ }^{4}$ \\ ${ }^{1}$ Department of Orthopedic Surgery, Tokyo Bay Urayasu Ichikawa Medical Center, Chiba, Japan \\ ${ }^{2}$ Department of Orthopedic Surgery, Doai Memorial Hospital, Tokyo, Japan \\ ${ }^{3}$ Department of Functional Joint Anatomy, Graduate School of Medical and Dental Sciences, Tokyo Medical and Dental \\ University, Tokyo, Japan \\ ${ }^{4}$ Department of Orthopedic Surgery, Graduate School of Medical and Dental Sciences, Tokyo Medical and Dental University, \\ Tokyo, Japan \\ Email: ^haruhikos@jadecom.jp
}

How to cite this paper: Shimura, H., Sato, T., Nimura, A., Wakabayashi, Y. and Fujita, K. (2019) Surgical Repair of Dorsal Capsulo-Ligamentous Complex for Dislocation of the Thumb Carpometacarpal Joint. Open Journal of Orthopedics, 9, 145-151. https://doi.org/10.4236/ojo.2019.98015

Received: June 20, 2019

Accepted: August 3, 2019

Published: August 6, 2019

Copyright (c) 2019 by author(s) and Scientific Research Publishing Inc. This work is licensed under the Creative Commons Attribution International License (CC BY 4.0).

http://creativecommons.org/licenses/by/4.0/

(c) (i) Open Access

\begin{abstract}
Background: Acute dislocation of the thumb carpometacarpal (CMC) joint is a rare and challenging injury, and its treatment remains controversial. The purpose of the study was to evaluate the clinical outcome after surgical repair of the dorsal capsulo-ligamentous complex. Materials and methods. Eight patients with a mean age of 39.8 years (range, 23 - 56 years) treated for the thumb CMC joint were treated in our hospital between 2008 and 2018. The ruptured dorsal capsulo-ligamentous complex was repaired using suture anchors in all patients, and the joint was immobilized with a Kirschner wire pinning and splint for 3 weeks. The clinical outcome was assessed by measuring the range of motion of the thumb CMC joint and the Japanese Society for Surgery of the Hand version of the Quick Disability of the Arm, Shoulder, and Hand (Q-DASH-JSSH) score. Results: The mean radial abduction of the thumb CMC joint was $66^{\circ}$, and the volar abduction was $63^{\circ}$. The mean Q-DASH-JSSH score was 4.8 (range, 0 - 15.6). There were no cases of infection, nerve disturbance, or osteoarthritis. Anatomical reduction was observed in seven patients at the final follow-up and in a rugby football player at 6 months of follow-up; however, subluxation of the thumb CMC joint at 12 months of follow-up due to reinjury was observed. Conclusion: Surgical repair of the dorsal capsulo-ligamentous complex with suture anchors is a reliable and simple treatment for acute unstable dislocation of the thumb CMC joint.
\end{abstract}

\section{Keywords}

Thumb Carpometacarpal Joint, Dislocation, Dorsal Capsulo-Ligamentous 
Complex, Surgical Repair, Suture Anchors

\section{Introduction}

Acute dislocation of the thumb carpometacarpal (CMC) joint is a rare, high-energy injury because of the strong capsulo-ligamentous complex structure. There are a few reports of this injury in the literature, and its optimal treatment remains controversial. Closed reduction and casting, closed or open reduction and percutaneous pinning, and open reduction and ligament reconstruction have been reported, some instability was found on follow up [1] [2] [3] [4]. The anterior oblique ligament was considered as a key stabilizer of the thumb CMC joint for many years [5]. However, Strauch et al. [6] found that the dorsoradial ligament was the primary restraint to dorsal dislocation in their cadaveric studies. Recently, some authors have introduced repair of the dorsal ligament using suture anchors based on the findings [7] [8] [9] [10]. The purpose of the study was to present our surgical procedures and evaluate the clinical outcome after repair of the dorsal capsulo-ligamentous complex using suture anchors.

\section{Methods}

Eight consecutive male patients (mean age, 39.8 years; range, 23 - 56 years) with unstable dorsal dislocation of the thumb CMC joint were treated between 2008 and 2018 in our hospital (Table 1). All acute traumatic dislocations of the thumb were immediately reduced, but the dorsal joint instability was recognized grossly. The ruptured dorsal capsulo-ligamentous complex was repaired using suture anchors in all patients, and the joint was immobilized with a 1.8 -mm Kirschner wire (K-wire) pinning and splint for 3 weeks. For one patient, 1.8-mm suture anchors (Mitek Mini anchor suture; Depuy-Synthes) were used, and, for the remaining seven patients, $1.0-\mathrm{mm}$ soft anchors (JuggerKnot soft anchor $1.0 \mathrm{~mm}$ Mini 2 - 0; Zimmer-Biomet) were used. The mean follow-up period was 10.7 months (range, 6 - 18 months). The grip strength was expressed as a percentage of the grip strength of the uninjured hand. In the grip strength analyses of right-handed patients, $10 \%$ was added to the left-side grip strength to compensate for hand dominance [11]. The range of motion (ROM) of the thumb CMC

Table 1. Summary of the cases.

\begin{tabular}{cccccc}
\hline Number & Age & Sex & Injury side & Cause of injury & Follow-up periods \\
\hline 1 & 32 & Male & Right & Playing rugby & 12 months \\
2 & 30 & Male & Right & Fall & 12 months \\
3 & 56 & Male & Right & Fall & 10 months \\
4 & 45 & Male & Left & Traffic accident & 18 months \\
5 & 43 & Male & Left & Fall from a motorcycle & 6 months \\
6 & 50 & Male & Right & Playing rugby & 12 months (subluxation) \\
7 & 23 & Male & Left & Traffic accident & 8 months \\
8 & 53 & Male & Right & Fall & 12 months
\end{tabular}


joint was measured in both the injured and the uninjured extremities with a goniometer, and the degree of volar and radial abduction was expressed as a percentage of the ROM of the uninjured joint. The clinical outcome was retrospectively assessed by measuring the Japanese Society for Surgery of the Hand version of the Quick Disability of the Arm, Shoulder, and Hand (Q-DASH-JSSH) score. Q-DASH-JSSH score is cross-culturally adapted and consists of an 11 items for self-assessment [12].

\section{Surgical Technique}

Surgery was performed with the patient under general anesthesia or brachial nerve plexus block by using an image intensifier. The thumb CMC joint was exposed by a dorsal approach or a modified Wagner's approach with a 4-cm curved skin incision. First, the abductor pollicis longus tendon was identified and retracted on the volar side. We found that the dorsal capsulo-ligamentous complex was ruptured in all patients. In seven patients, the dorsal capsulo-ligamentous complex was ruptured from the base of the first metacarpal bone, and, in the remaining one patient, it was ruptured from the trapezium. The dorsal capsulo-ligamentous complex had a strong and thick structure (Figure 1). The complex was repaired using two suture anchors after joint reduction (Figure 2). The thumb CMC joint was immobilized with a 1.8-mm K-wire pinning and splint for 3 weeks. The K-wire was removed at 3 weeks, followed by mild active and passive mobilization, and hard labor was prohibited for 2 months.

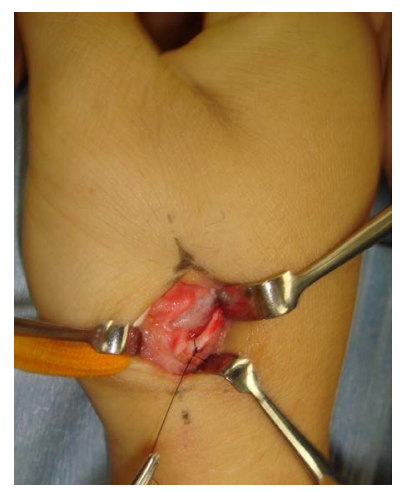

Figure 1. Intraoperative photograph showing avulsed dorsal capsule-ligamentous complex from the base of the right first metacarpal bone.

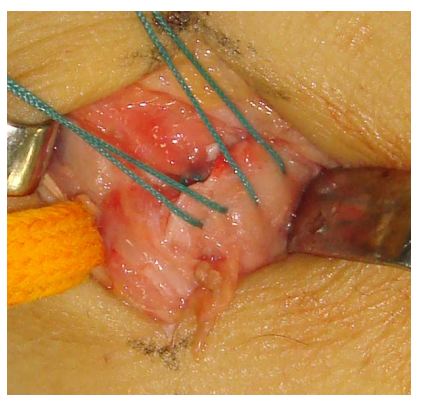

Figure 2. The dorsal capsule-ligamentous complex was repaired with suture anchors. 


\section{Results}

The mean volar abduction of the thumb CMC joint was $66^{\circ}(93.5 \%$ for the uninjured hand), and the mean radial abduction was $63^{\circ}$ (94.2\% for the uninjured hand). The mean Q-DASH-JSSH score was 4.8 (range, 0 - 15.6). The mean grip strength of the injured hand was $93.3 \%$ of that in the uninjured hand (Table 2). There were no cases of infection, radial superficial nerve or volar thenar median nerve branch disturbance, or early osteoarthritis. Anatomical reduction of the thumb CMC joint was observed in seven patients at the final follow-up. However, in a rugby football player, we found subluxation of the thumb CMC joint at 12 months of follow-up due to reinjury while playing rugby. In this patient, anatomical reduction was observed at 6 months of follow-up.

\section{Case Presentation}

A 30-year-old man presented after a 4-m fall from a ladder and reported left thumb pain. He was right-handed and worked as a planter. Anteroposterior and lateral hand radiographs showed an isolated dorsal dislocation of the thumb CMC joint (Figure 3). No fracture signs were identified by computed tomography. The dislocation of the thumb was immediately reduced, but fluoroscopic examination revealed gross instability of the thumb CMC joint. Surgery was recommended based on the patient's age and joint stability. Rupture of the dorsal capsulo-ligamentous complex from the base of the first metacarpal bone was observed. We repaired the dorsal capsulo-ligamentous complex using two soft anchors. Radiographs showed anatomical reduction of the thumb CMC joint at 12 months of follow-up (Figure 4). The volar abduction of the thumb CMC joint

Table 2. Clinical outcome.

\begin{tabular}{ccccc}
\hline Number & \%Grip strength & Volar abduction $\left({ }^{\circ}\right)$ & Radial abduction $\left({ }^{\circ}\right)$ & Q-DASH-JSSH \\
\hline 1 & 106.8 & 65 & 65 & 0 \\
2 & 81.8 & 65 & 70 & 4.5 \\
3 & 94.6 & 40 & 50 & 15.6 \\
4 & 90.0 & 60 & 60 & 0 \\
5 & 97.7 & 60 & 60 & 0 \\
6 & 93.3 & 80 & 75 & 13.6 \\
7 & 90.6 & 85 & 75 & 0 \\
8 & 91.2 & 75 & 50 & 4.5 \\
mean & 93.3 & 66.3 & 63.1 & 4.8 \\
\hline
\end{tabular}

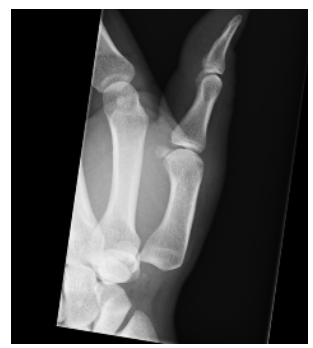

Figure 3. Preoperative radiograph of the thumb carpometacarpal joint showing an isolated dorsal dislocation. 


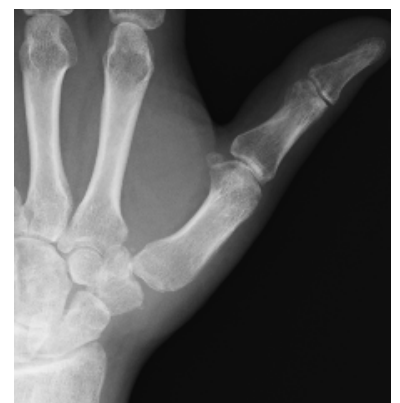

Figure 4. Postoperative radiograph of the thumb carpometacarpal joint showing good joint congruency.

was $65^{\circ}$, and the radial abduction was $70^{\circ}$. The Q-DASH-JSSH score was 4.5 . The patient currently has no thumb pain and has returned to his previous work.

\section{Discussion}

The thumb CMC joint is a saddle joint that is concave and convex on each side of the joint. The capsulo-ligamentous complex plays the principal role in providing stability due to the unique anatomy of the joint. Therefore, we must consider different treatment strategies than those for other digit joints. Eaton and Littler [5] considered the anterior oblique ligament as a key stabilizer of the joint that prevents dorsoradial subluxation of the thumb metacarpal. Therefore, they proposed reconstruction of the anterior oblique ligament, also known as the volar beak ligament. However, Strauch et al. [6] found that the dorsoradial ligament was the primary restraint to dorsal dislocation. In their cadaveric studies, the greatest degree of joint subluxation was present when all ligaments were intact and the dorsoradial ligament was sectioned. The least degree of joint subluxation was present when all ligaments of the thumb CMC joint, except the dorsoradial ligament, were sectioned. Ladd et al. [13] identified seven ligaments of the thumb CMC joint: three dorsal deltoid-shaped ligaments, two volar ligaments, and two ulnar ligaments. The dorsal ligament complex (mean thickness, $1.85 \mathrm{~mm}$ ) was thicker than the volar ligaments $(0.71 \mathrm{~mm})$ and the ulnar ligaments $(0.98 \mathrm{~mm})$. Based on this evidence, we have performed dorsal capsulo-ligamentous repair and not volar ligament repair. In our case series, the ruptured dorsal capsulo-ligamentous complex was identified and firmly repaired using suture anchors.

Treatment of the dislocation of the thumb CMC joint has been managed by three strategies [4]: closed reduction and casting, closed or open reduction and percutaneous pinning, and ligament reconstruction. Watt et al. [2] reported that closed reduction with casting and percutaneous pinning was applied with variable results in their series of 12 cases. Simonian et al. [3] reported that early ligament reconstruction resulted in a better outcome than that for closed reduction and pinning in a retrospective comparative study of 17 cases. Some case reports [14] [15] [16] [17] obtained good results after casting or percutaneous pinning. When the joint is stable after reduction, we agree with the conservative treat- 
ment or pinning. However, recurrent dislocation and revision surgery have also been reported after conservative treatment or pinning for gross instability of the thumb CMC joint. Recurrent dislocation and instability can lead to articular degeneration and chronic pain. In our study, all joints had gross instability after closed reduction. Recently some authors [7] [8] [9] [10] have reported repair of the ligament using suture anchors with more advanced medical devices. The advantages of ligament repair are that it is a simple procedure and is less invasive than ligament reconstruction. In addition, no tendon has to be sacrificed in repair with suture anchors in young and high-demand patients. Ligament reconstruction should be considered in cases of chronic dislocation.

The rugby football player in our study was injured again while playing rugby at 12 months postoperatively, and subluxation of the thumb CMC joint was observed on radiographic examination. The patient did not consent to revision surgery due to minor pain. At 6 months of follow-up, anatomical reduction and good function were observed. The patient understood the risk of reinjury from collision sports and returned to playing rugby football.

The limitations of this study are the small number of patients and the short follow-uptimes. Because the dislocation of the thumb CMC joint is such a rare injury, a large-scale multicenter study should be conducted to obtain evidence for this procedure. We consider that dorsal capsulo-ligamentous repair using suture anchors is a reliable and less-invasive treatment for acute, unstable dislocation of the thumb CMC joint in young patients.

\section{Conclusion}

Surgical repair of the dorsal capsulo-ligamentous complex with suture anchors is a reliable and simple treatment for acute unstable dislocation of the thumb CMC joint.

\section{Conflicts of Interest}

The authors declare no conflicts of interest regarding the publication of this paper.

\section{References}

[1] Shah, J. and Patel, M. (1983) Dislocation of the Carpometacarpal Joint of the Thumb. A Report of Four Cases. Clinical Orthopedics Related Research, 175, 166-169. https://doi.org/10.1097/00003086-198305000-00023

[2] Watt, N. and Hooper, G. (1987) Dislocation of the Trapezio-Metacarpal Joint. The Journal of Hand Surgery: British \& European Volume, 12, 242-245. https://doi.org/10.1016/0266-7681(87)90024-6

[3] Simonian, P.T. and Trumble, T.E. (1996) Traumatic Dislocation of the Thumb Carpometacarpal Joint: Early Ligamentous Reconstruction versus Closed Reduction and Pinning. Journal of Hand Surgery American Volume, 21, 802-806. https://doi.org/10.1016/S0363-5023(96)80195-X

[4] Bosmans, B., Verhofstad, M.H. and Gosens, T. (2008) Traumatic Thumb Carpometa- 
carpal Joint Dislocations. Journal of Hand Surgery American Volume, 33, 438-441. https://doi.org/10.1016/j.jhsa.2007.11.022

[5] Eaton, R.G. and Littler, J.W. (1973) Ligament Reconstruction for the Painful Thumb Carpometacarpal Joint. The Journal of Bone and Joint Surgery American Volume, 55, 1655-1666. https://doi.org/10.2106/00004623-197355080-00010

[6] Strauch, R.J., Behrman, M.J. and Rosenwasser, M.P. (1994) Acute Dislocation of the Carpometacarpal Joint of the Thumb: An Anatomic and Cadaver Study. The Journal of Bone and Joint Surgery American Volume, 19, 93-98. https://doi.org/10.1016/0363-5023(94)90229-1

[7] Fotiadis, E., Svarnas, T., Lyrtzis, C., Papadopoulos, A., Akritopoulos, P. and Chalidis, B. (2010) Isolated Thumb Carpometacarpal Joint Dislocation: A Case Report and Review of the Literature. Journal of Orthopaedic Surgery and Research, 5, 16. https://doi.org/10.1186/1749-799X-5-16

[8] Okita, G., Anayama, S., Sato, N. and Haro, H. (2011) Surgical Reconstruction Using Suture Anchor for Dislocation of the Carpometacarpal Joint of the Thumb: A Case Report. Archives of Orthopaedic and Trauma Surgery, 131, 225-228. https://doi.org/10.1007/s00402-010-1122-3

[9] Ansari, M.T., Kotwal, P.P. and Morey, V.M. (2014) Primary Repair of Capsuloligamentous Structures of Trapeziometacarpal Joint: A Preliminary Study. Journal of Clinical Orthopaedics and Trauma, 5, 185-192. https://doi.org/10.1016/j.jcot.2014.09.009

[10] Lahiji, F., Zandi, R. and Maleki, A. (2015) First Carpometacarpal Joint Dislocation and Review of Literatures. Archives of Bone Joint Surgery, 3, 300-303.

[11] Crosby, C.A., Wehbe, M.A. and Mawr, B. (1994) Hand Strength: Normative Values. The Journal of Bone and Joint Surgery American Volume, 19, 665-670. https://doi.org/10.1016/0363-5023(94)90280-1

[12] Imaeda, T., Toh, S., Wada, T., Uchiyama, S., Okinaga, S., Kusunose, K. and Sawaizumi, T. (2006) Validation of the Japanese Society for Surgery of the Hand Version of the Quick Disability of the Arm, Shoulder, and Hand (Quick DASH-JSSH) Questionnaire. Journal of Orthopaedic Science, 11, 248-253. https://doi.org/10.1007/s00776-006-1013-1

[13] Ladd, A.L., Lee, J. and Hagert, E. (2012) Macroscopic and Microscopic Analysis of the Thumb Carpometacarpal Ligaments: A Cadaveric Study of Ligament Anatomy and Histology. The Journal of Bone and Joint Surgery American Volume, 94, 1468-1477. https://doi.org/10.2106/JBJS.K.00329

[14] Uchida, S., Sakai, A., Okazaki, Y., Okimoto, N. and Nakamura, T. (2001) Closed Reduction and Immobilization for Traumatic Isolated Dislocation of the Carpometacarpal Joint of the Thumb in Rugby Football Players. Two Case Reports. American Journal of Sports and Medicine, 29, 242-244. https://doi.org/10.1177/03635465010290022201

[15] Jakobsen, C.W. and Elberg, J.J. (1988) Isolated Carpometacarpal Dislocation of the Thumb. Case Report. Scandinavian Journal of Plastic and Reconstructive Surgery and Hand Surgery, 22, 185-186. https://doi.org/10.3109/02844318809072395

[16] Khan, A.M., Ryan, M.G. and Teplitz, G.A. (2003) Bilateral Carpometacarpal Dislocations of the Thumb. The American Journal of Orthopedics, 32, 38-41.

[17] Mueller, J.J. (1986) Carpometacarpal Dislocations: Report of Five Cases and Review of the Literature. The Journal of Bone and Joint Surgery American Volume, 11, 184-188. https://doi.org/10.1016/S0363-5023(86)80048-X 